\section{SEM Processing Of Blood Samples}

Sara E. Miller, Duke University Medical School

A long time ago, we did some work on RBC's from muscular dystrophy patients. We found that the cells are very sensitive to $\mathrm{pH}$, salt concentration, glass, and other things (maybe including the moon???). Low pH, increased salt, especially $\mathrm{NaCl}$, and sitting in a glass tube or on a glass slide unfixed would cause echinocyte formation.

We got around the problem by drawing the blood and then IMMEDIATELY dropping (drop by drop) blood samples into $1 \%$ glutaraldehyde in phosphate buffer, $\mathrm{pH} 7.4$ (not PBS). You can use a Maunsbach formula, where $0.135 \mathrm{M} \mathrm{PO}_{4}$ gives 300 milliosmoles, or $0.1 \mathrm{M} \mathrm{PO}_{4}$ plus sucrose (try 1.8 $\%$ sucrose). Test the osmolarity of the buffer; it should be $300 \pm 10$ or so. The final osmolarity of the buffered fix will be 400 milliosmoles: 300 from the buffer and 100 from the glutaraldehyde! The fix should be room temperature (not cold) when the blood is added. It can be stored refrigerated.

Only about 2-3 drops of blood should be put into $10 \mathrm{~mL}$ fix. Gently invert several times to mix but don't shake vigorously. Let the blood cells sit in the tube, and they will settle to the bottom. Also, a drop of suspension may be put onto a collagen-coated coverslip, and the cells allowed to settle out for an hour (covered and in a moist chamber).

They can be dehydrated by gently adding increasing percentages of ethanol to one side of the coverslip with a Pasteur pipette while removing the old from the other side with another pipette. If you just put enough solution on the coverslip to mound up (without overfilling it) you can keep a bubble of cells/fix/ethanol on it without its running off the edge.

Critical point dry the sample (be sure to place the cell side of the coverslip away from the jet of liquid $\mathrm{CO}_{2}$ in the drier).

To make collagen-coated coverslips:

Mix $1 \%$ collagen in water: you may have to heat gently to dissolve, and it will take a while to go into solution. Filter if it still has big chunks of undissolved material. With a Pasteur pipette, place collagen onto coverslips, rounding it up, but not letting it run over the side. Allow them to sit for 10-20 minutes, then remove the collagen, which can be reused for other coverslips. Dip coverslips gently into deionized water and air-dry.

You can mark the coverslips by notching a small nick into the edge with forceps (do this before you collagen-coat, because some of them will break in the process):

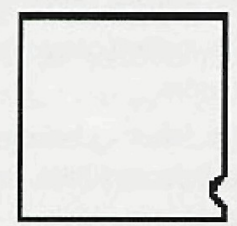

Then if they become flipped over, you will know on which side the cells are. Just remember where your mark is, e.g., bottom right.

I don't remember which references contain the method, but try these:

Miller, S.E., A.D. Roses and S.H. Appel. 1975. Erythrocytes in human muscular dystrophy, Science 188:1131.

Roses, A. D., S. H. Appel, D. A. Butterfield, S. E. Miller and D. B. Chesnut. 1975. Specificity of biochemical and biophysical tests in Duchenne and myotonic muscular dystrophy, carrier states and congenital myotonia. Trans. Amer. Neurol. Assoc. 100:131-134.

Roses, A.0., M.J. Roses, S.E. Miller, K.J. Hull, Jr. and S.H. Appel. 1976. Carrier detection in Duchenne muscular dystrophy, New Engl. J. Med. 294:193-198.

Miller, S.E., A D. Roses and S. H. Appel 1976. Scanning electron microscopy studies in muscular dystrophy. Arch. Neurol. 33:172-174.

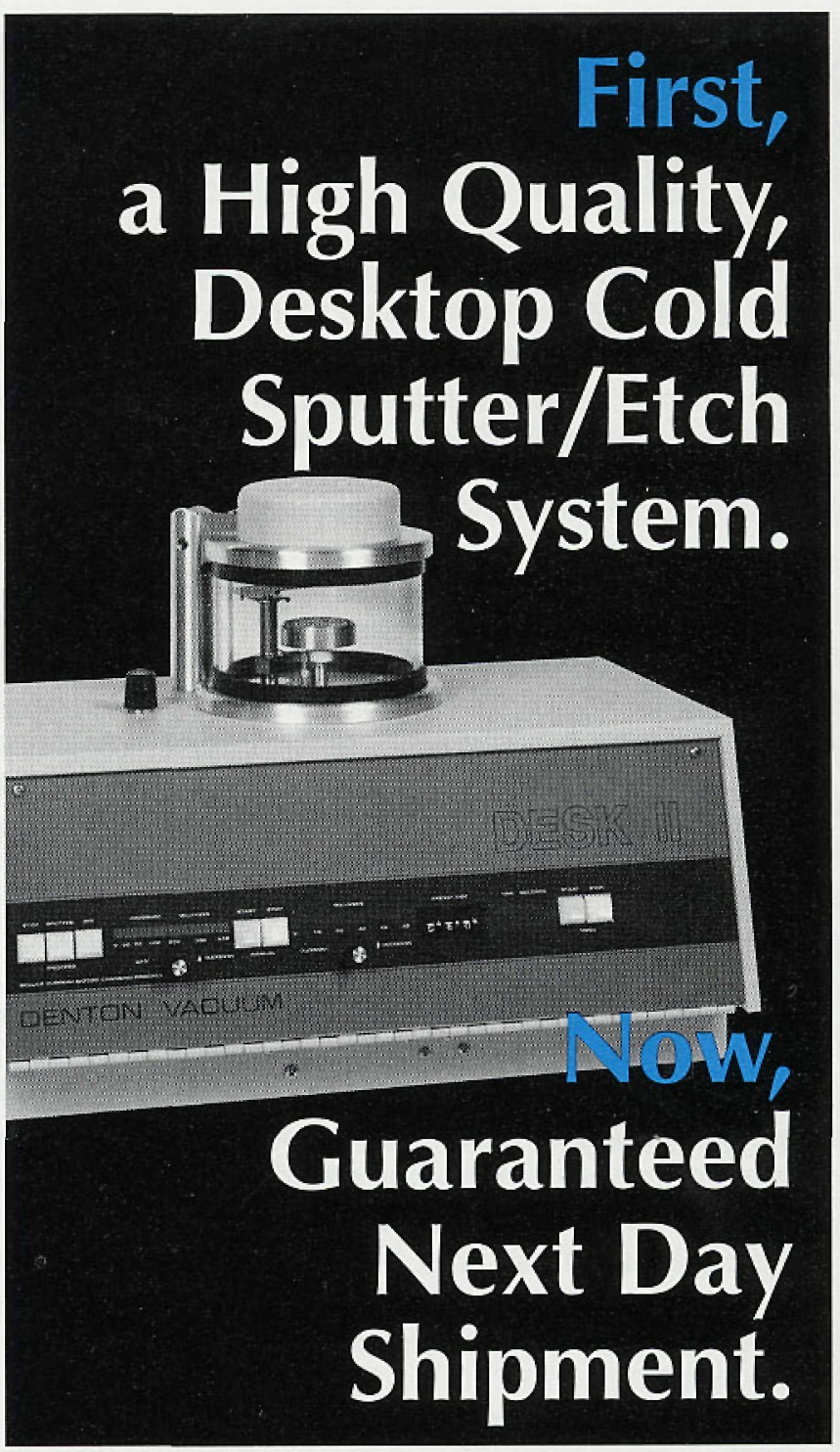

Order Denton's standard Desk II System by noon today and get guaranteed shipment tomorrow or Denton pays the freight.

The Desk II produces uniform, conductive, fine grain $\mathrm{Au} / \mathrm{AuPd}$ coatings in under three minutes and is the highest quality desktop system available.

\section{The Desk // features:}

\section{Automatic or manual operation}

Easy to read digital vacuum and current gauges

Optional carbon evaporation accessory

For more information, look no further than Denton... where both Quality and Delivery invite comparison.

\section{DENTON VACUUM INC.}

1259 North Church St. Moorestown, NJ 08057 USA

Tel: (609) 439-9100

FAX: (609) 439-9111

t MADE IN AMERIGA

Next day shipment must be requested at time of order.

Decades of Experience In EM Specimen Prep Equipment 